Fecha de entrega: 15 de julio de 2010

Fecha de aprobación: 3 de noviembre de 2010

\title{
LAS IDEAS EDUCATIVAS DE JOSÉ CELESTINO MUTIS Y BOSIO THE EDUCATIVES IDEAS OF JOSÉ CELESTINO MUTIS Y BOSIO
}

\author{
Alberto Isaac Rincón Rueda*
}

\section{Resumen}

José Celestino Mutis y Bosio es considerado uno de los sabios más significativos que llegaron desde la península ibérica a la Nueva Granada en la Colonia. Se dedicó a la medicina, a la difusión de las ciencias útiles, a la ilustración, al estudio de la flora y la fauna del país, labor que se vio reflejada en su trabajo como docente. Impulsó las ideas educativas ilustradas, que pretendían superar el atraso educativo y cultural que vivían los habitantes de la Nueva Granada por un sistema educativo basado en la escolástica, que no permitió el desarrollo de las ciencias. Las ideas educativas ilustradas de Mutis propusieron el estudio de las matemáticas, la química, la medicina en el mundo académico colonial. Ideas que se denotan en los trabajos de la Expedición Botánica.

\section{Palabras clave}

Ideas, educación, ciencias útiles, matemáticas, ilustración.

\section{Abstract}

José Celestino Mutis y Bosio is considered one of the most notable sages to arrive from the Iberian Peninsula to New Granada in colonial times. He was devoted to medicine, the diffusion of useful sciences, Illustration, and the study of flora and fauna of the country. These practices were reflected in his work in education. He boosted educational ideas from

* Docente de la Facultad de Filosofía y Letras de la Universidad Santo Tomás y del I.E.D. Fernando Mazuera Villegas, Bogotá, D.C. 
the Age of Enlightenment that aimed at overcoming educational backwardness prevalent in New Granada, due to an educational system based on Scholasticism, which did not allow the development of sciences. The Enlightened educational ideas of Mutis proposed the study of mathematics, chemistry and medicine in the colonial academic environment. These ideas are denoted in the works of the Botanic Expedition.

\section{Keywords}

Ideas, education, useful sciences, mathematics, illustration.

\section{En los doscientos años de la muerte del ilustre sabio gaditano}

\section{La llegada de Mutis a la Nueva Granada}

Mutis ha sido considerado como uno de los científicos más importantes en la Nueva Granada en el período colonial, egresado de la Universidad de Sevilla en donde se graduó de bachiller; luego desarrolló estudios de medicina que lo llevaron a obtener el título de médico; asimismo, había estudiado gramática, filosofía y teología; con una inclinación académica por las matemáticas, las ciencias naturales y en especial por la botánica, consolidó el estudio de estas ciencias en la ciudad de Cádiz y luego en Madrid en 1757, allí perfeccionó sus labores en ciencias exactas y en 1760 continuó sus estudios en el Jardín Botánico de Soto de Migas Calientes bajo la dirección de Bornades, "de este modo el célebre gaditano se instruyó en la nueva escuela lineana, que más tarde había de divulgar con entusiasmo en la América española al mismo tiempo con las matemáticas, la física y la astronomía” (Gredilla,1982: 32).

Mutis decide acompañar como médico de cabecera al marqués de la Vega de Amijo don Pedro Messia de la Cerda y Cardona quien fuera nombrado Virrey de la Nueva Granada, la idea del gaditano era el estudiar la geografía, la flora y la fauna de América, llegó a Cartagena de Indias el 29 de septiembre de
1760, donde inició un recorrido por la costa atlántica y luego tomó el camino que lo conduciría a la capital del Virreinato de la Nueva Granada a orillas del río Magdalena, recorrió poblaciones como Mompox, Tamalameque, San Pablo, Canta Gallo y Honda, llegando el 24 de febrero de 1761 a Santafé de Bogotá.

La idea de Mutis era dedicarse al estudio de la flora y la fauna neograndinas, idea que se truncó por espacio de dos años, en los que se dedicó de lleno a su profesión de médico, ya que eran muy pocos los que habían en la capital del Virreinato en esa época, como lo manifestó él mismo, "la novedad del nuevo médico, junto a la escasez de facultativos, cortó el vuelo de mis ideas, de día en día me vi empeñado en la asistencia de muchos enfermos (cuyas observaciones reservo aparte) y en los del mayor cuidado, unos cuidados de tan grande importancia, con el trabajo material de pasar de casa en casa, me quitaron todo aquel ocio que pide un estudio serio" (Mutis, 1761, cap. II, párr. 1), la labor de médico para él fue extenuante, con largas caminatas, con cambios de clima que sufría para cumplir con la labor de visitar enfermos, lo que no le permitía desarrollar su labor investigativa científica, así lo refería en su diario de observaciones:

...es imponderable la multitud de obstáculos que continuamente ocurren o interrumpen mis tareas literarias en 
asuntos de historia natural. Apenas me queda tiempo para ocuparme en estas materias, ni sirviéndome de poco consuelo la justa desconfianza con que sospecho frustrados mis proyectos (1761, cap. II, párr. 1).

Con los recursos económicos obtenidos del sueldo continuó su trabajo de historia natural. Mutis destinó algunas horas de su tiempo para dar lecciones de matemáticas y filosofía newtoniana, pretendía aportar a los cambios que necesitaba la Nueva Granada, en especial en el campo de la educación, ayudó a la juventud con ideas renovadoras que aportaran a la educación en las ciencias aplicadas desde el aula, a partir de las teorías de Isaac Newton y de los científicos del viejo continente desconocidos en el mundo académico de la Nueva Granada, lo que permitió tener otra visión del conocimiento a la de la escolástica tradicional.

El científico español encaminó sus trabajos a conseguir resultados, "por lo mismo toda la vida de Mutis se convirtió en continuo enseñar y enderezar voluntades, un perpetuo interés por la juventud, para trascender hacia el futuro de la Nueva Granada y aportar más al bienestar de quienes lo rodeaban" (Pérez, 1998: 76). Muchos de sus discípulos fueron los protagonistas de la independencia nacional.

Mutis en su casa dictó clases de matemáticas, el momento más significativo en la enseñanza fue:

Cuando tomó posesión de la cátedra de matemáticas en el Colegio del Rosario de Santafé de Bogotá, hacia fines de 1766 , siendo esta la primera vez que se oyeron lecciones de tales ciencias en el virreinato de la Nueva Granada desde su conquista y (siendo) el medio más adecuado para instruir a la juventud bogotana y hacerle cobrar adicción por el estudio de las ciencias (Gredilla, 1982: 38).

Con esta cátedra se quería dar a conocer los nuevos avances científicos desarrollados en Europa e introducirlos en el sistema educativo colonial. Estos planteamientos se sustentaron en las ideas de la Ilustración y pretendían, de manera fundamental, lo útil, el progreso, el desarrollo de la industria, la minería y la educación, lo cual era prioridad para el monarca español Carlos III.

\section{Las ideas educativas de José Celestino Mutis}

Las ideas educativas que propuso tenían que ver con el estudio de las matemáticas y con la enseñanza de las nuevas ciencias en los colegios de la capital del Virreinato, las nuevas propuestas científicas iniciadas por el sabio gaditano ocasionaron rupturas a través de la enseñanza al dar a conocer las revoluciones científicas de la época, además de ideas y conceptos sobre la naturaleza y el cosmos, lo que ocasionó conflictos y polémicas con los sectores académicos tradicionales que estaban marcados por la escolástica y la tradición religiosa, asimismo, con políticos que estaban ajenos a los cambios que se estaban presentando en Europa y en España particularmente.

El 13 de marzo de 1762 se realizó el acto de inauguración de la Facultad de Matemáticas a cargo del doctor José Celestino Mutis, las autoridades del Colegio Mayor de Nuestra Señora del Rosario de Santafé de Bogotá afirmaron:

...habiéndose propuesto por don José Celestino Mutis, médico de cámara del excelentísimo señor 
Baylio Fray don Pedro Messia de la Cerda, actual virrey de estos reinos, la intención con que se hallaba de enseñar las partes de matemáticas por lo útil y necesarias que se estimaban para varios destinos y que con tales en el presente siglo se cursan en las principales universidades de Europa (Hernández, 1976: 212).

Con la apertura de la cátedra de matemáticas se desarrolló la idea de poner como eje las ciencias, la educación en la Nueva Granada, lo que permitió el avance del conocimiento en la juventud de la capital del Virreinato y, en especial, entre los estudiantes del Colegio Mayor de Nuestra Señora del Rosario: “...que se planifique la lección de matemáticas por su conducencia y facilidad con que puede conseguirse enseñando en un país donde se encuentran tantos ingenios aptos para su instrucción" (1976: 212). Con una oración pronunciada en latín, el sabio Mutis manifestó lo útil, lo honroso y lo necesario para el impulso del conocimiento, las ciencias necesarias para el hombre, el estudio de las matemáticas y para otorgar a la educación cambios significativos, donde las ideas matemáticas fueran el centro de la nueva academia.

Mutis, en el discurso de apertura del curso de matemáticas en el Colegio Mayor de Nuestra Señora del Rosario, manifestó:

...la utilidad de una ciencia parece ser el motivo que más obliga a cultivarla con algún empeño; y siendo tan manifiesta para el mundo las utilidades de las matemáticas, no es de extrañar que muchos hombres de competencia hayan rodado en esta parte por todos los siglos con mayor fortuna que en las otras ciencias (Hernández, 1983: 39).
Las matemáticas desde el mundo de los griegos fueron fundamentales para el desarrollo del conocimiento y la cultura. Europa se detiene cuando es invadida por los bárbaros entre quienes las ciencias, el conocimiento y las letras pasaron a un segundo plano y el progreso tiene dificultades. Según Mutis: “....a fines del siglo pasado y principios del presente, renacieron las matemáticas con tales ventajas a aquellos primeros tiempos que es muy notable la diferencia" (1983: 39), en realidad los estudios de las matemáticas aumentaron, lo que permitió el avance en la ciencias y difundieron el conocimiento y de las ciencias útiles en el territorio del Virreinato; poniendo en práctica la educación matemática en la que se formaron jóvenes científicos, que trabajaron en la Expedición Botánica y muchos de ellos fueron protagonistas de las gestas de independencia, decía Mutis: "el nacimiento, progreso, revolución y actual estado de las matemáticas piden un estudio continuado por muchos años y una abundantísima biblioteca abastecida de manuscritos, memorias, instrucciones y libros antiguos y modernos" (1983: 40).

Mutis quería dar a conocer a la sociedad santafereña y a los jóvenes en su cátedra de matemáticas la utilidad de las ciencias y su estudio, ya que muchos creían que no era útil, y como las matemáticas se relacionan con otras ciencias para dar buenos resultados, útiles en el conocimiento, los jóvenes deberían aprenderla tal como lo dijo: “... todos los hombres deberían instruirse en las matemáticas pues bien es cierto que rústicos, ciudadanos, plebeyos, cortesanos, militares, artífices, sabios, seculares, eclesiásticos, todos en una palabra, de cualquier condición y estado deberían aplicarse a un estudio tan útil" (1983: 40). Esto permitiría a los hombres recibir el conocimiento de las matemáticas que aplicarían luego en la vida cotidiana 
para la solución de problemas, incluso este conocimiento científico matemático permitía a las personas conocer y contemplar la obra del Creador y llegar donde surge lo creado; así, el hombre debería venerar al Creador porque "cuando Dios creó al mundo, esta máquina tan maravillosa, que no acabaremos de admirar bastantemente, parece haberse formado entonces el alto designio de poner en práctica las leyes matemáticas" (1983: 41). Afirmaba también que al crear Dios el mundo "todo lo dispuso en número, peso y medida con un orden y establecimientos tan constantes que permanecerán hasta cierto día" (1983: 41).

Todo está determinado por movimientos, el mundo creado por leyes que los hombres han querido saber, se preguntó Mutis: “¿a qué grado de perfección no elevarían sus conocimientos los que se instruyesen en las matemáticas?" (1983: 42). Todo esto permitiría que el conocimiento fuera más verídico, transparente con las otras ciencias, "las matemáticas por lo general son rechazadas por aquellos hombres que han sido instruidos en letras y en disciplinas que tenían que ver con lo sagrado, siendo las matemáticas llave maestra de todas las otras ciencias" (1983: 42) y que tienen relación con las demás ramas de la literatura, cultivar las matemáticas en las instituciones educativas del Nuevo Reino de Granada ayudaría a superar el atraso educativo y crear caminos para el florecimiento de las ciencias, como lo propuso Mutis: “...la necesidad de las matemáticas para el cultivo, adelantamiento y esplendor de todas las ciencias. El estudio de la lógica, llave de las ciencias y bellas artes, suele preceder a la instrucción de las demás facultades" (Hernández, 1976: 216). En el estudio de las matemáticas la lógica desempeña un papel importante, pues entrega las reglas fundamentales para el ordenamiento del pensamiento matemático y las demás ciencias. Era fundamental que los jóvenes estudiaran geometría, pues permite llegar a demostraciones, la física era imprescindible en las ideas del sabio Mutis, y en su estudio de estas ciencias se podía utilizar el método sintético y el analítico.

Con estas propuestas se pretendió superar la escolástica, la lógica aristotélica, la filosofía tradicional, al tener presente los planteamientos de las filosofías modernas, derrotando el peripato, al demostrar que las ciencias modernas se han desarrollado porque han recibido aportes fundamentales de la filosofía, como es el caso de "la física por el grande Newton y por sus esclarecidos secuaces Gravesande, Musschenbroek y Mollet, entre otros, igualmente acreedores a las mayores alabanzas" (1976: 218). La física en el siglo XVIII logró avances que llevaron a que esta disciplina científica se consolidara con los estudios y descubrimientos de Isaac Newton y otros, dando un verdadero impulso al estudio de la naturaleza y logrando lo útil, sacándole beneficio para el ser humano. Mutis con sus ideas educativas trató de capacitar a los estudiantes en los nuevos conocimientos que brindaba la ciencia.

Sus ideas no reñían con lo religioso, ni con la fe, decía: “...no son las matemáticas ocupación extraña a un teólogo sobre el testimonio que nos ofrecen santos venerables y varones ilustrados aplicados gloriosamente a unos estudios tan útiles en todos los siglos" (1976: 220). Estudios útiles como las matemáticas no estaban en contra de la teología y las Sagradas Escrituras, el papa Benedicto XIV había permitido adelantar estudios de física y ciencias matemáticas en la Universidad de Bolonia, por esto,

...la preocupación principal de $\mathrm{Mu}-$ tis era demostrar la utilidad de las matemáticas, no sólo en el campo 
de las ciencias sino también para la vida cotidiana; de ahí que con tanta insistencia le propusiera a todo el mundo dedicarse al estudio de esta ciencia porque según él, nunca era inútil el estudio de estas disciplinas para el servicio de la religión, el rey y la patria (Soto, 1989: 34).

Era en este ámbito que quería desarrollar sus ideas, afianzadas en autores como Isaac Newton, Melchor Cano, Gravesande, Musschenbroek, desarrollando en cada curso de matemáticas, los principios de filosofía natural de Newton: física, aritmética, trigonometría, geometría y elementos de mecánica.

\section{Consolidación de las ideas de Mutis en la educación en el Nuevo Reino de Granada}

Para instaurar su propuesta educativa, el sabio José Celestino Mutis, en 1764, presentó un trabajo sobre elementos de la filosofía natural, que contenía los principios de la física demostrada por las matemáticas y confirmados con observaciones y experiencias, dispuesto a instruir a la juventud en la doctrina de la filosofía newtoniana en el Colegio de Nuestra Señora del Rosario de Santafé de Bogotá, así, insistía constantemente en su trabajo con los jóvenes para superar el atraso cultural que se vivía en la Colonia, era fundamental el desarrollo de las ciencias y el "...conocimiento útil y agradable de los efectos naturales y de sus causas, han merecido siempre la atención de los sabios" (Hernández, 1982: 44). Por tanto, en sus preocupaciones educativas resaltó como prioridad el estudio de la naturaleza que permitiría el desarrollo económico y educativo, se estudiarían las plantas, los animales y los minerales, para esto era importante la instrucción de los jóvenes en disciplinas como química, matemáticas, astronomía, anatomía; y para que estas propuestas dieran resultado se tendría que partir de la física experimental que permitió el progreso de las ciencias, las artes, la filosofía natural que estudiaba la naturaleza y cuyo objeto "es describir los fenómenos de la naturaleza, descubrir sus causas, exponer sus relaciones y el orden del universo" (1982: 45), además la filosofía de la naturaleza intenta consolidar la religión natural y, junto con la filosofía moral, llevar al hombre a un conocimiento superior que le permitiría llegar al autor de la naturaleza, al Creador del Universo, como lo decía: "estudiar la naturaleza es lo mismo que dirigirse a conocer las obras maravillosas de aquel soberano Creador, que se deja conocer en parte por las cosas visibles" (1982: 46). El conocimiento conduce al Creador de la naturaleza pero han existido falsos sistemas de la física que han llevado al hombre al ateísmo y a opiniones que no corresponden al Creador, dando paso a las supersticiones. El hombre tiene que superar estas situaciones, la incertidumbre del conocimiento.

Mutis fundamentó los planteamientos de Isaac Newton, consideró que no se podría hacer un sistema completo de la naturaleza, pero de todos modos era fundamental consultar a la naturaleza, seguir los pasos y sacar de ella las cosas ocultas por medio de la experiencia, las observaciones, incluso valiéndose de la geometría. Newton planteó la teoría de la gravedad, sus ideas fueron duramente criticadas "no es novedad que la filosofía newtoniana haya pasado por tantas opiniones" (1982: 56), por aquellos formados en los viejos sistemas de pensar, que no dan resultados positivos, o por la escolástica que no permitía el progreso y el desarrollo de nuevas ideas educativas y científicas para alcanzar un mayor progreso.

Mutis invitaba a cambios profundos en los que los jóvenes serían los beneficiados al dar a conocer los preceptos de las ciencias que se desarrollaban en Occidente, al decir: "pero no 
se ha podido impedir que la juventud, como menos impresionaba en los falsos sistemas, haya dejado de obrar con mucho gusto, el estudio de la verdadera filosofía" (1982: 57), con el conocimiento de las ciencias útiles y el desarrollo de ellas se lograría el progreso en la Nueva Granada.

En su afán de afianzar las ideas educativas ilustradas que estaban ligadas al conocimiento de las ciencias útiles, en los centros educativos de las colonias y en especial en los colegios de Santafé. Mutis realizó una sustentación del sistema heliocéntrico de Copérnico en el Colegio Mayor de Nuestra Señora del Rosario, en honor del excelentísimo virrey don Manuel Guirior y doña María Ventura Guirior (1773).

\begin{abstract}
Al inicio de su disertación felizmente conducido por la oculta mano de la Providencia acaba de llegar vuestra excelencia a este reino constituido en los últimos alientos de la miserable fortuna en que ha vivido; y acaba de ver vuestra excelencia lo que tal vez no había pensado: las rentas atrasadas, el comercio perdido, las artes abandonadas y las ciencias abatidas (Archivo General de la Expedición Botánica del Nuevo Reino de Granada).
\end{abstract}

Mutis le solicitó al virrey que le diera impulso a una educación donde "....se digne promover las ciencias, ello producirá sabios talentos que aspirando a la sólida gloria de hacerse útiles a la religión, al rey y a la patria" (Archivo General de la Expedición Botánica del Nuevo Reino de Granada: 2). En las ciudades del antiguo continente desde las cátedras de teología y filosofía se atacaba el ateísmo y la filosofía da un profundo significado al mundo de la sabiduría, a la ilustración, al entendimiento por medio de la observación, la experiencia y la razón al superar la física arábiga.

Además, propuso:

...ya es tiempo, señor excelentísimo, de romper el hielo y que, animada la juventud por el buen gusto que domina a vuestra excelencia, emprenda un nuevo viaje literario, saliendo finalmente, aunque bien cansado, de los campos estériles de la física aristotélica para convalecer el ánimo en los americanos presos de la filosofía newtoniana (Archivo General de la Expedición Botánica del Nuevo Reino de Granada: 4).

Creía necesario que la educación desarrollara cambios para que los jóvenes educados en los colegios y en las universidades tuvieran la posibilidad de conocer los avances de las ciencias y la filosofía, y los desarrollos en estos campos de la modernidad; las ideas educativas de Mutis se relacionaban con estas ciencias, era su preocupación que los jóvenes las aprendieran y aplicaran en el desarrollo de estas tierras, este conocimiento basado en las teorías de Newton, y de Copérnico con su sistema heliocéntrico, dio bases para saber del Universo. Sustentaba Mutis con estas afirmaciones lo siguiente: "los jóvenes americanos iban recibiendo la filosofía moderna, por la utilidad grande que nos prometíamos, por la declarada voluntad del monarca que así lo manda y por la protección del sabio Virrey que promovió las ciencias" (Archivo General de la Expedición Botánica del Nuevo Reino de Granada: 6).

De esta manera, Mutis quería acabar con los viejos sistemas educativos al impulsar ideas modernas de ciencia y educación, y enfrentándose a conceptos y al medio académico atrasado que no quería cambios significativos, por eso quería ideas nuevas en 
la educación y en el conocimiento; consideraba su alegato necesario "para defender en un acto literario los principios más curiosos con que se halla ilustrada la filosofía moderna que desea ver introducida en sus alumnos" (Archivo General de la Expedición Botánica del Nuevo Reino de Granada: 8) y el conocimiento científico útil.

Por eso los colegios tenían que introducir las nuevas ciencias y la filosofía moderna, como el Colegio Mayor de Nuestra Señora del Rosario de Santafé, estas ideas no reñían con la religión, la Iglesia romana no se oponía a los estudios copernicanos ni a los conflictos teológicos.

... las conquistas de la investigación ignoraban que el monarca liberal Carlos III había ordenado el estudio de Newton, que vale decir Copérnico y que por aquel innovador guiaba Mutis a sus discípulos camino de la ciencia y la libertad (Hernández, 1957: 151).

Los cambios educativos encaminados al estudio de las ciencias se desarrollaron en las universidades de Valladolid, Salamanca y Alcalá, donde se trabajó la astronomía, siguiendo las ideas del astrónomo inglés Jaime Fergusson. Por tanto, la educación, las ciencias que debían ser trabajadas en los colegios de la Nueva Granada. Esta era la preocupación de Mutis.

\section{Planes de estudio propuestos por Mutis}

Mutis para consolidar sus ideas educativas propone una metodología para la enseñanza de las matemáticas en el Colegio Mayor de Nuestra Señora del Rosario, afirmaba que "todo el artificio de las matemáticas, su certidumbre y solidez consiste en el admirable orden de que usan las matemáticas para enseñar sus dogmas" (Hernández, 1957: 1), es una disciplina severa que permite la resolución de problemas, usa la demostración para buscar resultados verdaderos en el que las pruebas son fundamentales, "ha merecido esta ciencia, por la solidez que le es muy particular, calificar todo método exacto en cualquier materia que sea y este modo de proceder de los matemáticos es lo que se llama método geométrico" (1957: 2). El método geométrico se fundamenta en reglas de carácter general que permiten consolidar esta disciplina, cuya finalidad es encontrar la verdad partiendo de tres ideas que son fundamentales: primero, de las ideas más sencillas a las más compuestas; segundo, de términos no ambiguos y, tercero, afirmando las verdades por medio de proposiciones y demostraciones. Las matemáticas tienen definiciones, axiomas, postulados, demostraciones que conducen a que las matemáticas sean una disciplina práctica puntual, "que si este orden tan riguroso se hallará observado puntualmente en otras ciencias serían mucho menos los atrasos que hubieran padecido" (1957: 5). Con estas reflexiones quería aportar al desarrollo matemático en los estudios escolares de la Nueva Granada y al desarrollo científico académico del Nuevo Reino.

En 1787 Mutis presentó en el Colegio Mayor de Nuestra Señora del Rosario un plan provisional para la enseñanza de las matemáticas, apoyado por el Arzobispo Virrey Antonio Caballero y Góngora, este plan "se dirigía a propagar entre la juventud americana los conocimientos de una ciencia necesaria al bien del Estado en el mismo grado supremo en que se consideren por todo el mundo sabio las ciencias útiles" (Mutis: 83). Con este plan, y las ideas contenidas, buscaba el abandono de la ignorancia, la superación del atraso cultural, el progreso que se tenía que dar en la industria, artes, comercio y agricultura, y que eran las bases fundamentales para el sustento del hombre y de la sociedad que 
busca el progreso, una nación que quiera salir adelante tiene que permitir el desarrollo de los estudios de matemáticas, ya que "aquellas forman los matemáticos de profesión y estos los sabios puramente aficionados a extender en lo posible sus conocimientos para su ilustración en su respectiva carrera" (Mutis: 84). Los participantes en esta cátedra tenían que ser los interesados en las matemáticas como ciencia, el maestro tenía que dar los fundamentos teóricos y científicos, todo esto permitió el surgimiento de los estudios de las matemáticas en la Nueva Granada, se siguió a Volffio, quien formó grandes matemáticos. La escuela juega un papel fundamental, "de cuya instrucción en lo más esencial y de su noticia en lo puramente de curiosidad e ilustración no deben defraudarse nuestros jóvenes en los elementos de la escuela" (Mutis: 86).

España se preocupó por desarrollar las matemáticas en la Real Escuela de San Fernando, dirigida por el matemático don Benito Bails, por su contenido científico se necesitó tiempo y dedicación, en la Nueva Granada por falta de libros de matemáticas, decía Mutis: "se obliga a tolerar la mal introducida costumbre de escribir en las aulas con detrimento del tiempo precioso y otros perjuicios que justamente han desterrado de las escuelas pésima ocupación y sólo subsistirá mientras llegan estos ejemplares" (Mutis: 88).

El plan que contenía las ideas educativas matemáticas comprendía de tres a cuatro años, en el primero se trabajarían lecciones privadas en vacaciones de los escolares para fortalecer la enseñanza; durante el período académico se trabajaría en las horas de la mañana dos horas y media y en la tarde hora y media, empleados para la escritura, repeticiones y demostraciones, ejercicios, preguntas de las lecciones antecedentes. En el caso de la "enseñanza pública el aula hará observar al catedrático la regularidad y la buena crianza que siendo preceptos recomendados en toda escuela, se han hecho ya característicos del Colegio de Nuestra Señora del Rosario" (Mutis: 90), para que el plan diera los resultados esperados era necesario que de la península se trajeran los instrumentos necesarios para el estudio de las matemáticas, los estudiantes tenían que tener para el trabajo reglas y compás además del papel apropiado para el caso, se trabajarían los tratados que conformaban la obra de Benito Bails. Los alumnos destacados serían los que participaran en los actos públicos, en las clases se debían nombrar de monitores.

Estas ideas educativas para la enseñanza de las matemáticas que proponía Mutis, lograron ser escuchadas por las autoridades peninsulares, y el 6 de julio de 1787, por orden real, se establecen las facultades de matemáticas en el Colegio Mayor de Nuestra Señora del Rosario de Santafé, nombrándose profesor al doctor don Fernando de Vergara, quien sería sustituto del doctor Mutis "al restablecer la cátedra de matemáticas cuyo estudio es tan importante para las demás ciencias, especialmente las naturales" (Archivo General de la Expedición Botánica del Nuevo Reino de Granada); pero la cátedra no tuvo estudiantes por lo cual no se obtuvieron los resultados esperados, en 1791 los estudiantes del Colegio de San Bartolomé le solicitan a los gobernantes del Virreinato se les nombre un profesor que les enseñe elementos de "física, matemáticas, botánica e historia natural, donde pudieran aprender las teorías de Descartes y Newton" (A.U.N.C.). Estos estudiantes se oponían a las teorías de Goudin, lo mismo que los colegiales del Rosario, esto demuestra que las ideas de Mutis dieron frutos en los establecimientos educativos de Santafé, en los que se preparaba la élite criolla, que quería cambios significativos en la educación, allí se desarrollaban las ciencias útiles y las ideas ilustradas, sin embargo, ante los nuevos conceptos que beneficiarían la educación del Virreinato existió una fuerte oposición que atacó las enseñanzas de la nueva ciencia. 
En 1802 Jorge Tadeo Lozano, a petición de Mutis, continúa con la cátedra de matemáticas del Colegio Mayor de Nuestra Señora del Rosario, en 1806 la cátedra es dirigida por el ingeniero Bernardo Anillo y en 1809 es nombrado profesor de matemáticas Francisco José de Caldas, además fue importante profesor de matemáticas José Félix de Restrepo en el Colegio Seminario de Popayán entre 1782 y 1812, en Medellín en 1813 y luego en Santafé en el Colegio de San Bartolomé.

\section{Los planes de estudio de Mutis en medicina}

En 1791 la cátedra de medicina en Santafé de Bogotá estaba vacante, el virrey don Pedro Messia de la Cerda solicitó al rey que nombrara a José Celestino Mutis como catedrático.

...y en este caso parece correlativo y justo que el mismo don José Celestino Mutis ejerza el protomedicato durante su obtención de la cátedra que pasará después sucesivamente a los que la granjearon por oposición y se conseguirá el alivio de tener médicos de suficiencia (Barras, 1941: 32).

Mutis no aceptó la cátedra de medicina y continuó en su trabajo de matemáticas e historia natural, pero preocupado por el desarrollo de la medicina propone dos cátedras, una de medicina y la otra de anatomía, expresó sus ideas sobre la enseñanza de la medicina en la propuesta que presentó en 1781 al arzobispo virrey don Antonio Caballero y Góngora, en la que se propuso la cátedra de medicina, siguiendo los criterios y metodologías de las universidades más adelantadas de la península ibérica, se contaría con un teatro anatómico donde se harían las disecciones de animales, los estudiantes tendrían la posibilidad de obtener esqueletos y cuerpos artificiales, contarían, según el plan, con dos médicos españoles quienes se dedicarían a las cátedras de química y botánica.

El gaditano consciente de la necesidad de los estudios de medicina en los centros educativos del Virreinato, presentó un informe sobre el estado de la medicina en estas tierras el 3 de junio de 1801, en el que demuestra la escasez de verdaderos profesores de esta disciplina y sí la existencia de curanderos. Por tanto, era necesario establecer la cátedra para superar los males que aquejaban a la población y “...al establecimiento de las cátedras y protomedicato en la capital para la enseñanza pública y arreglo de las tres ramas de la profesión señalándoles sus buenas dotaciones" (Mutis: 45). Su ideal, al establecer la cátedra de medicina, era formar buenos médicos y cirujanos, con buena práctica, con un gran bagaje académico, "perpetuar sucesivamente la enseñanza de modo que dentro de pocos años se pueda propagar el mismo beneficio a las menores poblaciones" (Mutis: 47), era necesario que el gobierno fijara un establecimiento permanente para el desarrollo de la cátedra de medicina, de la enseñanza y los maestros que se dedicaran a la formación de los futuros médicos, por tanto,

...bastan por ahora las ocho cátedras del primer establecimiento permanente y siempre fijas en los sucesivo, sin exclusión de otras dos o tres que se podrían fundar en adelante, con el fin de ampliar la enseñanza de los conos auxiliares y relativos a la salud pública (Mutis: 48).

Los estudiantes tenían que recibir la formación en las ocho cátedras que tenían que ver con las matemáticas, la física en todas sus ramas, la anatomía, cuya enseñanza se practicaría en cadáveres, la medicina teórica, la medicina práctica, que era conocer todas las enfermedades, otra cátedra era la doctrina hipocrática o ciencia del contenido de la biblia 
médica, también la medicina clínica que era la práctica y por último la cátedra de botánica que era la de historia natural, se complementaba con las de farmacia y química; para el desarrollo de este plan se contaría con catedráticos escogidos en España. Las cátedras de anatomía y de cirugía práctica debían ser separadas y ocupadas por estudiantes bien preparados en medicina.

Para que las ciencias médicas se desarrollaran con plenitud, Mutis presentó un plan general de estudios según la realidad del país, plan que se implementaría en el Colegio Mayor de Nuestra Señora del Rosario, esta propuesta académica para la formación de médicos comprendía cinco cursos, que implicarían cinco años, al cabo de los cuales se podrían graduar en la Facultad; los estudiantes recibirían las cátedras de anatomía teórica y práctica en el hospital; instituciones médicas, patología general y particular, la doctrina hipocrática, los estudios prácticos serían en el hospital durante tres años, con esta práctica los médicos recibirían y revalidarían la licencia para curar. La preocupación de Mutis por la enseñanza de las ciencias médicas era su prioridad, los profesores estaban dedicados a trabajar por el alivio y el consuelo del dolor en las mujeres, y la cátedra de medicina y su enseñanza en el Nuevo Reino no se consolidó con la apertura de los estudios, por eso la idea educativa que tenía era la de consolidarlos, por lo que proponía,

...dejando ya en el olvido la suerte infeliz que le ha tocado en el dilatado curso de más de dos siglos, se lisonjea la capital con las próximas y bien fundadas esperanzas de la creación de las cátedras de medicina y demás ciencias subalternas, a imitación de los ventajosos establecimientos hechos en España (Archivo de la Real Expedición Botánica del Nuevo Reino de Granada, legado 50-62: 10).
Con la apertura y planes de la cátedra de medicina se tenía que mejorar la enseñanza, desterrando de las aulas los antiguos métodos de enseñanza como el peripatético arábigo, que perjudicaron el progreso de las ciencias naturales, como los planteamientos de partido de las escuelas opuestas, lo cual según Mutis "sería la última desgracia de este Reino para el colmo de sus miserias mantener todavía las rancias preocupaciones de nuestros mayores" (Mutis: 64); el objeto de la medicina y sus estudios es conservar la salud y restablecer al hombre de las enfermedades. Se completan los estudios preparatorios con introducción al latín, griego y la filosofía racional con lógica y ética, los estudios estarán solidificados con autores como Winslow, la célebre patología de Gombius, los tratados del Gortex y los tratados del ilustre profesor de Montpellier Sauvayus, en estudios de tiempo completo.

\section{Propuesta de Mutis para la cátedra de química}

Consciente Mutis, de la necesidad de mejorar la educación en la Nueva Granada y en el desarrollo de sus ideas educativas, propuso, el 9 de noviembre de 1801, el establecimiento de un laboratorio de química con su cátedra respectiva, adjunta a la Facultad de Medicina del Colegio Mayor de Nuestra Señora del Rosario, proponiendo como primer catedrático a Jorge Tadeo Lozano. Esta cátedra era un espacio de desarrollo de las ciencia naturales y la medicina, ello justificaba su creación. Esta cátedra no sólo estaba dirigida a los estudiantes de medicina y a los profesores de las ciencias de la salud, sino a otros estudiantes como lo afirmó él mismo: "son ellas unas ciencias más generales en que pueden igualmente instruirse los cursantes de otras profesiones y demás jóvenes aficionados, según la inclinación de su genio y promover de algún modo la felicidad pública" (Hernández, 1957: 2). La química es fundamental porque permite la investigación de la naturaleza y 
las propiedades de ella y los cuerpos, las naciones desarrolladas le han dado importancia a esta ciencia y han establecido la cátedra pública y privada de la química, además era fundamental porque "instruidos en la química podían promover los conocimientos especulativos y operaciones prácticas de la mineralogía" (1957: 3).

El éxito de esta cátedra estaba en la enseñanza pública, ciencia desconocida en la Nueva Granada, y que traería beneficios para el trabajo de la minería,

...las proposiciones de esta ciencia no debe retardar el establecimiento premeditado, porque sus ideas irán aficionando a la juventud a ejecutar por sí misma las operaciones más sencillas, además de poderse así conseguir un más que mediano conocimiento de la mineralogía, mientras se logra la aplicación de fondo proyectando el general establecimiento de cátedras (1957: 4).

Esto permitió el avance de la ilustración en el Reino y el desarrollo de las ideas educativas que se fundamentaron en las ciencias útiles que eran vitales para el adelanto de estas tierras.

Mutis fue el director de la Expedición Botánica en la Nueva Granada y quería con ésta el estudio de la naturaleza, para sacar provecho, incluso estudió sobre las bebidas fermentadas, entregando un informe el 13 de mayo de 1771, fue un hombre ilustrado que quería el cambio para la Nueva Granada y sus habitantes con una buena educación.

\section{A modo de conclusión}

Las ideas educativas de José Celestino Mutis y Bosio, fueron esenciales para la difusión de la ilustración y las ciencias útiles en los territorios coloniales. Con su trabajo se buscó alcanzar el desarrollo de la Nueva Granada y seguir las políticas ilustradas españolas del monarca Carlos III en el mundo colonial, lo que implicaba, para la época, transformar el sistema educativo.

\section{Referencias}

A.U.N.C. (1791). Los colegiales de filosofía del Colegio de San Bartolomé solicitan se les conceda pagar a sus expensas un catedrático que les enseñe filosofía moderna y principios de matemáticas. Santafé: Colonia Fondo Milicias y Marina. T. 128, folios 200-201.

Barras, F. (1941) Noticias y documentos referentes al insigne gaditano de la Universidad de Sevilla, don José Celestino Mutis. En Anales de la Universidad Hispalense Sevilla, año IV, núm. 1.

Gredilla, F. A. (1982). A José Celestino Mutis: complemento a la historia extensa de Colombia. Bogotá: Academia Colombiana de Historia - Plaza \& Janés.

Hernández, G. (1957). Crónica del muy ilustre Colegio Mayor de Nuestra Señora del Rosario en Santafé de Bogotá (libro segundo). Bogotá: Edición Centro.

Hernández, G. (1976). Documentos para la historia de la educación en Colombia. (t. III) (compilación) . Bogotá: Editorial Kelly.

Hernández, G. (Comp.) (1982). Pensamiento científico y filosófico de José Celestino Mutis. Bogotá: Ediciones Fondo Cultural Cafetero.

Hernández, G. (1983). Escritos cientificos de don José Celestino Mutis. Bogotá: Ediciones Instituto Colombiano de Cultura Hispánica - Ediciones Kelly. 
Hernández, G. (s. f.). Documento original conservado.

Mutis, J. C. (s. f.). Diario de observaciones del año 1761. Archivo del Colegio Mayor de Nuestra Señora del Rosario, vol. 10, folio 83 .

Mutis, J. C. (s. f.). Manuscrito. En Colección Pineda, vol. 191. Bogotá: Biblioteca Nacional - sala de manuscritos.
Pérez, E. (1998). José Celestino Mutis: su vida y su obra. Bogotá: Ediciones Fondo FEN Colombia.

Real Jardín Botánico de Madrid. Archivo General de la Expedición Botánica del Nuevo Reino de Granada.

Soto, D. (1989). Mutis, filósofo, educador. Bogotá: Universidad Pedagógica Nacional. 